\title{
Einstellungskriterien in der Inneren Medizin im Lichte der neuen ÄAppO
}

\section{Engagement election criteria in internal medicine in the light of the new legislation for medical education in Germany ( $\ddot{A}$ AppO)}

- Stefanie Geisenhof ${ }^{1} \cdot$ Thomas Shiozawa $^{2} \cdot$ Simon Mayer $^{1} \cdot$ Martin R. Fischer $^{1}$

\section{Text}

Einleitung: Seit Oktober 2003 ist die neue Approbationsordnung in Kraft [1]. Im Zuge dieser Reform werden in den klinischen Fächern Einzelnoten vergeben, die Ende 2006 erstmals im neuen 2. Staatsexamen erscheinen werden. Die Bedeutung dieser Noten ist bezüglich ihres Einflusses auf Einstellungskriterien bisher unklar.

Methoden: Mittels eines elektronischen Fragebogens wurden Einstellungskriterien auf einer fünfstufigen numerisch verankerten Likert-Skala (1: trifft überhaupt nicht zu; 5: trifft voll zu) nach ihrer Wichtigkeit bewertet [2]. Der Fragebogen wurde im Mai 2006 an 8500 Mitglieder der deutschen Gesellschaft für Innere Medizin (DGIM) versandt. Der Rücklauf lag bei 436 Fragebögen (5,1\%): 79 Chefärzte, 70 Oberärzte, 132 Fachärzte sowie 155 Assistenten in Weiterbildung. 46,3\% der Befragten haben Personalverantwortung.

Ergebnisse: Als besonders wichtig für die Entscheidung zur Einstellung eines ärztlichen Mitarbeiters nach Studienabschluss wurden die persönliche Kenntnis durch ein PJ-Tertial (Mittelwert 4,16; SD 1,3) oder Doktorarbeit $(3,83 \pm 1,2)$ sowie die persönliche Empfehlung durch einen Kollegen $(3,49 \pm 1,1)$ eingeschätzt. Ebenfalls wichtig wurden soziales Engagement und Zusatzqualifikationen gewertet.

Bezüglich der Notenvergabe nach alter ÄAppO waren die Gesamtnote im Staatsexamen $(3,49 \pm 1,1)$ und die Note im 3. Staatsexamen $(3,40 \pm 1,1)$ besonders wichtig.
Hinsichtlich der zukünftigen Notenvergabe wurden die Einzelnoten von 38,3\% der Befragten als wichtiger als das Ergebnis im 2. Staatsexamen eingestuft. 38,7\% werteten die Note 4 (ausreichend) in der Inneren Medizin als Ausschlusskriterium bei der Bewerberselektion.

Diskussion: Als Einstellungskriterien wurden die persönliche Kenntnis des Bewerbers, eine persönliche Empfehlung, soziales Engagement sowie Zusatzqualifikationen wichtiger als die Noten im Staatsexamen gewertet. Die fachbezogenen Einzelnoten spielen in Relation zur Note im 2. Staatsexamen eine untergeordnete Rolle.

\section{Korrespondenzadresse:}

- Dr. med. Martin R. Fischer, Medizinische Klinik-Innenstadt, Klinikum der Universität München, Ziemssenstr. 1, 80336 München, Deutschland, Tel.: 089/5160-2159, Fax: 089/5160-2366 fischer.martin@med.uni-muenchen.de

\section{Literatur:}

[1] Bundesministerium für Gesundheit und Forschung. Approbationsordnung für Ärzte vom 27. Juni 2002. Bundesgesetzbl. 2002;44:2405-2435.

[2] Casus.net [homepage im Internet]. Fragebogen. München: Arbeitsgruppe Medizindidkatik; 2006. Zugänglich unter http://eval.casus.net/survey/index.php?sid=33 